\title{
Research on Control Strategy of Double Local-fans Monitoring System Based on DSP
}

\author{
ZHANG Hong ${ }^{\mathrm{a}}$ \\ ${ }^{a}$ School of Computer and Communication Engineering, Weifang University, Weifang 261061, China
}

\begin{abstract}
For fault diagnosis and data processing in coal mine safety production, this paper gives a kind of data processing method based on Grubbs's criterion and weighted data fusion. The method is based on intelligent detecting system and data fusion technology, it eliminates measurement data containing mistake errors by Grubbs's criterion first and then determines weight number in weighted data fusion, at last, it calculates fusion estimate to carry out fault diagnosis. The measured data verification shows that this kind of data fusion algorithm has advantages of more accurate results, simple algorithm and strong practicality, etc.
\end{abstract}

Index Terms: Double Local-Fans; Monitoring and Control; Gas Superscale; Data Fusion

(C) 2011 Published by MECS Publisher. Selection and/or peer review under responsibility of the International Conference on E-Business System and Education Technology

\section{Introduction}

Our country's coal safety production accidents take place frequently and there have been many very severe coal mine gas accidents in recent years. For coal mine safety production, this paper puts forward a kind of fault diagnosis and data processing method in double local-fans monitoring system based on DSP to solve the problem how to handle working face, air inlet, return airway and hybrid return airway after over-standard gas concentration in coal mine production.

\section{Selection of Data Preprocessing Method}

This paper is a study on the method of underground working face gas fault diagnosis and how to handle the collected underground gas concentration data is very important. The data preprocessing method of this system mainly refers to carrying out mistake error processing of detection data of gas sensor to eliminate uncertain values in initial measurement values of a single sensor, thereby improving the accuracy and repeatability of measurement results and obtaining higher real-time measurement results.

Mistake error refers to a kind of error which doesn't tally with the fact obviously and it is a serious perversion of measurement results. This kind of mistake error is not permitted in detecting systems, so Corresponding author:

E-mail address: ${ }^{a}$ zhanghongwf@ sina.com 
detection data containing mistake errors should be eliminated to ensure the accuracy of detection results. The processing methods of mistake errors mainly include Letts criterion and Grubbs's criterion[1,2], etc.

Description of Letts criterion: Letts criterion is one of common methods for judging mistake errors, but it is built on the foundation that the measurement time $\mathrm{n}$ tends to infinity, when the measurement time is a finite value, especially when the measurement time $\mathrm{n}$ is relatively small, it is not applicable.

Description of Grubbs's criterion: carry out equal precision detection of a measured sample many times and obtain a set of measurement data $X_{1}, X_{2}, \ldots, X_{n}$, suppose that the set of data has been arranged from small to large, namely $X_{1} \leq X_{2} \leq \ldots \leq X_{n}$ and $X_{i}(i=1,2, \ldots, n)$ obeys normal distribution. Calculate the mean value $\bar{X}$, residual error $v_{i}$ and approximate error before mistake errors are eliminated $\sigma^{\prime}$, then find out the exact distribution of statistics $g$. Therefore, after significant level $\alpha$ (generally, $\alpha=0.05$ or $\alpha=0.01$ ) is given, the critical value of Grubbs statistics $g_{0}(n, \alpha)$ can be found out by looking up Table [2], namely $p\left[g \geq g_{0}(n, \alpha)\right]$ $=\alpha$ is a small probability event and it should not appear when $X_{i}$ obeys normal distribution.

Measure Grubbs statistics $g_{i}$ corresponding to top value $X_{i}(i=1$ or $n)$, if $g_{i} \geq g_{0}(n, \alpha)$, it is considered that the distribution of statistics $g_{i}$ has a significant difference and the corresponding measurement data $X_{i}$ contains mistake errors and it is considered doubtful, so it should be eliminated. If $g_{i}<g_{0}(n, \alpha)$, it is considered that the corresponding $X_{i}$ doesn't contain mistake errors.

For the data whose measurement time is little, Grubbs's criterion has a high accuracy of eliminating the data containing mistake errors and it can program by computer very easily. In this system, the sampling number of gas sensor for gas concentration is finite, suppose that carry out data processing after sampling eight times. And therefore, Grubbs's criterion is more suitable for data preprocessing in this system.

\section{Data Processing Algorithm Based on Grubbs's Criterion}

In this system, there are four kinds of main gas concentration sensors for detecting corresponding gas concentrations, namely $\mathrm{T}_{1}$-tunneling head gas concentration sensor, $\mathrm{T}_{2}$-tunneling return air gas concentration sensor, $\mathrm{T}_{3}$-hybrid return air gas concentration sensor and $\mathrm{T}_{4}$-fan air inlet gas concentration sensor. Coal mine safety production regulations require that when the concentrations at $\mathrm{T}_{1}$ and $\mathrm{T}_{2}$ exceed $1.5 \%$, it is considered that gas concentrations are over standard and it is required to cut off the working face; when the concentration at $\mathrm{T}_{3}$ exceeds $1.5 \%$, it is considered that gas concentration is over standard and it is required to cut off local fans and working face to form a closed lock; when the concentration at $\mathrm{T}_{4}$ exceeds $1.5 \%$, it is considered that gas concentration is over standard and it is required to cut off local fans and working face to form a closed lock.

\subsection{Elimination of mistake errors}

The sampling values of $T_{1}, T_{2}, T_{3}$ and $T_{4}$ for gas concentration during a period of time are shown as Table 1 .

Table 1. Sampling Values of $\mathrm{T}_{1}, \mathrm{~T}_{2}, \mathrm{~T}_{3}$ and $\mathrm{T}_{4}$ During a Period of Time

\begin{tabular}{ccccccccc}
\hline $\boldsymbol{T}$ & $\mathbf{1}$ & $\mathbf{2}$ & $\mathbf{3}$ & $\mathbf{4}$ & $\mathbf{5}$ & $\mathbf{6}$ & $\mathbf{7}$ & $\mathbf{8}$ \\
\hline $\mathbf{T}_{\mathbf{1 i}}(\boldsymbol{\%})$ & 1.36 & 1.38 & 1.34 & 1.38 & 1.39 & 1.28 & 1.35 & 1.36 \\
$\mathbf{T}_{\mathbf{2}}(\boldsymbol{\%})$ & 1.28 & 1.30 & 1.44 & 1.32 & 1.34 & 1.27 & 1.26 & 1.32 \\
$\mathbf{T}_{\mathbf{3} i}(\boldsymbol{\%})$ & 1.13 & 1.15 & 1.12 & 1.18 & 1.14 & 1.25 & 1.16 & 1.17 \\
$\mathbf{T}_{\mathbf{4} i}(\boldsymbol{\%})$ & 0.45 & 0.43 & 0.43 & 0.42 & 0.44 & 0.46 & 0.54 & 0.45 \\
\hline
\end{tabular}


Carry out Grubbs elimination of the data collected by gas sensor $\mathrm{T}_{1}$. Arrange the eight sets of gas concentrations detected by $\mathrm{T}_{1}$ from small to large: $T_{16}, T_{13}, T_{17}, T_{11}, T_{18}, T_{12}, T_{14}$ and $T_{15}$.

Calculate the mean value of measurement 8 times $\bar{T}_{1}=1.3550$. Calculate the residual error and the corresponding residual errors are shown as Table 2.

Table 2. Residual Errors of $\mathrm{T}_{1}$

\begin{tabular}{ccccccccc}
\hline $\boldsymbol{i}$ & 1 & 2 & 3 & 4 & 5 & 6 & 7 & 8 \\
\hline $\boldsymbol{v}_{\mathbf{1} i}$ & 0.005 & 0.025 & 0.015 & 0.025 & 0.035 & 0.075 & 0.005 & 0.025 \\
\hline
\end{tabular}

Calculate the approximate error before mistake errors are eliminated $\sigma^{\prime}=0.0346$.

It can be seen from Table 2 that $\left|v_{16}\right|=\left|v_{1 i}\right|_{\max }=0.075$ is doubtful. Here, let significant level $\alpha=0.05, g_{0} \quad$ ( 8 , $0.05)=2.03$ can be got and then $g_{0}(8,0.05) \sigma^{\prime}=2.03 \times 0.0346=0.0702$.

Because $\left|v_{16}\right|=\left|v_{1 i}\right|_{\max }=0.075>g_{0}(8,0.05) \sigma^{\prime}=0.0702$, it can be judged that $T_{16}=1.28 \%$ is the measurement value containing mistake error and it should be eliminated.

After $T_{16}$ is eliminated, the rest seven data and residual errors are shown as Table 3.

Table 3. Table of Residual Data And Errors of $\mathrm{T}_{1}$

\begin{tabular}{cccccccc}
\hline $\boldsymbol{i}$ & $\mathbf{1}$ & $\mathbf{2}$ & $\mathbf{3}$ & $\mathbf{4}$ & $\mathbf{5}$ & $\mathbf{6}$ & $\mathbf{7}$ \\
\hline$T_{1}^{\prime}$ & 1.36 & 1.38 & 1.34 & 1.38 & 1.39 & 1.35 & 1.36 \\
$\boldsymbol{v}_{\mathbf{1}}$ & -0.0057 & 0.0143 & -0.0257 & 0.0143 & 0.0243 & -0.0157 & -0.0057 \\
\hline
\end{tabular}

Calculate the mean value of the rest seven data $\overline{T_{1}^{\prime}}=1.3657$ and calculate the approximate error $\sigma^{\prime}=0.0181$. And then $g_{0}(7,0.05) \sigma^{\prime \prime}=1.94 \times 0.0181=0.0351>\left|v_{1 i}^{\prime}\right|_{\max }=\left|v_{13}^{\prime}\right|=0.0257$. It can be judged that there isn't data containing mistake error in the rest seven data.

Similarly, for the rest three sets of data $\left(\mathrm{T}_{2}, \mathrm{~T}_{3}\right.$ and $\left.\mathrm{T}_{4}\right)$, eliminate the data containing mistake error by Grubbs's criterion [2]. The data after four sets of data are processed by Grubbs's criterion is shown as Table 4.

Table 4. Data after Mistake Errors Are Eliminated By Grubbs's Criterion

\begin{tabular}{cccccccc}
\hline $\boldsymbol{T}$ & $\mathbf{1}$ & $\mathbf{2}$ & $\mathbf{3}$ & $\mathbf{4}$ & $\mathbf{5}$ & $\mathbf{6}$ & $\mathbf{7}$ \\
\hline $\boldsymbol{T}_{\mathbf{1}}(\boldsymbol{\%})$ & 1.36 & 1.38 & 1.34 & 1.38 & 1.39 & 1.35 & 1.36 \\
$\boldsymbol{T}_{\mathbf{2}}(\boldsymbol{\%})$ & 1.28 & 1.30 & 1.32 & 1.34 & 1.27 & 1.26 & 1.32 \\
$\boldsymbol{T}_{\mathbf{3}}(\boldsymbol{\%})$ & 1.13 & 1.15 & 1.12 & 1.18 & 1.14 & 1.16 & 1.17 \\
$\boldsymbol{T}_{\mathbf{4}}(\boldsymbol{\%})$ & 0.45 & 0.43 & 0.43 & 0.42 & 0.44 & 0.46 & 0.47 \\
\hline
\end{tabular}

\subsection{Calculation of mean value and divided difference after mistake errors are eliminated}

After the calculation of eliminating the data containing mistake errors, the rest data doesn't contain mistake errors, which provides convenience for the subsequent calculation. According to the data in Table 4 calculate 
the mean value $\bar{T}_{i}$ of $\mathrm{T}_{1}, \mathrm{~T}_{2}, \mathrm{~T}_{3}$ and $\mathrm{T}_{4}$ after mistake errors are eliminated, the residual error $v_{i}$ after mistake errors are eliminated and the divided difference of each set of data after mistake errors are eliminated can be calculated by $v_{i}$. The mean values and divided differences after mistake errors are eliminated are shown as Table 5 .

Table 5. Mean Value and Divided Difference of $\mathrm{T}_{1}, \mathrm{~T}_{2}, \mathrm{~T}_{3}$ and $\mathrm{T}_{4}$ after Mistake Errors Are Eliminated

\begin{tabular}{ccccc}
\hline Category & $\mathbf{1}$ & $\mathbf{2}$ & $\mathbf{3}$ & $\mathbf{4}$ \\
\hline $\boldsymbol{T}_{\mathbf{1}}(\boldsymbol{\%})$ & 1.3567 & 1.2986 & 1.1500 & 0.44 \\
$\boldsymbol{\sigma}_{\boldsymbol{i}}(\boldsymbol{\%})$ & 0.0183 & 0.0297 & 0.0216 & 0.0141 \\
\hline
\end{tabular}

\subsection{Data comparison before and after mistake errors are eliminated}

According to the data in Table 1 calculate the mean value $\bar{T}_{i}$ of $\mathrm{T}_{1}, \mathrm{~T}_{2}, \mathrm{~T}_{3}$ and $\mathrm{T}_{4}$ before mistake errors are eliminated, the residual error ${ }^{v_{i}}$ before mistake errors are eliminated and the divided difference of each set of data before mistake errors are eliminated can be calculated. The mean values and divided differences before mistake errors are eliminated are shown as Table 6.

It can be seen by comparing Table 5 with Table 6 that the divided differences of data in Table 6 before mistake errors are eliminated are significantly greater than the divided differences in Table 5 after mistake errors are eliminated, which shows the accuracy of data after mistake errors are eliminated is high and proves the validity of eliminating mistake error data by Grubbs's criterion, it lays a good foundation for the accurate measurement of the system.

Table 6. Mean Value and Divided Difference of $\mathrm{T}_{1}, \mathrm{~T}_{2}, \mathrm{~T}_{3}$ and $\mathrm{T}_{4}$ before Mistake Errors Are Eliminated

\begin{tabular}{ccccc}
\hline Category & $\mathbf{1}$ & $\mathbf{2}$ & $\mathbf{3}$ & $\mathbf{4}$ \\
\hline $\bar{T}_{t}(\%)$ & 1.3567 & 1.2986 & 1.1500 & 0.44 \\
$\sigma_{i}(\%)$ & 0.0183 & 0.0297 & 0.0216 & 0.0141 \\
\hline
\end{tabular}

\section{Intelligent Weighted Data Fusion Processing Method}

\subsection{Weighted data fusion algorithm based on Grubbs's criterion}

There are many related data fusion algorithms based on data fusion technology [3] and these algorithms are more favorable for improving and enhancing the accuracy of detection. Combined with the practical situation of the system, this section studies how to carry out weighted data fusion processing of various sensor data after mistake errors are eliminated.

\section{1) Description of weighted data fusion:}

Suppose that in a multi-sensor detection system, use $n$ sensors to carry out sampling detection of a detection object and get a set of data $X_{1}, X_{2}, \ldots, X_{n}$, different data has a corresponding weight, under the optimum 
condition of least total mean square deviation, find the corresponding data in adaptive mode according to measured values to make $\hat{X}$ after fusion optimal. The weighted data fusion model is shown as Fig 1 .

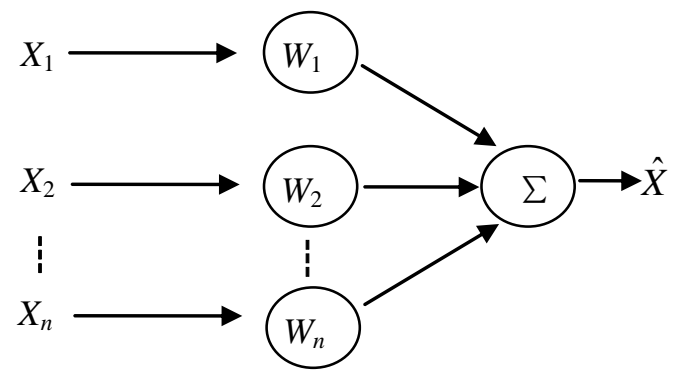

Fig. 1. Weighted Data Fusion Model

In Fig $1, W_{1}, W_{2}, \ldots, W_{n}$ are weights corresponding to data $X_{1}, X_{2}, \ldots, X_{n}$ as well as data outputs after weighted fusion, it can be seen from the fig that the value after fusion is

$$
\hat{X}=\sum_{i=1}^{n} W_{i} X_{i}
$$

\section{2) Description of weighted data fusion algorithm based on Grubbs's criterion:}

Suppose that use a type I sensor to detect a detection object many times and get a set of data $X_{1}, X_{2}, \ldots, X_{L}$, after mistake error data is eliminated by Grubbs's criterion, the arithmetic mean value of measurement results of type I sensor is got as the initial value $\overline{X_{I}}$ of data fusion. Similarly, the mean values of measurement results of type II, III ,.., $\mathrm{N}$ sensor $\overline{X_{I I}}, \overline{X_{I I I}}, \ldots, \overline{X_{N}}$ can be got as the initial values of data fusion. At last, the weighted fusion value got is

$$
\hat{X}=\sum_{i=I}^{N} W_{i} X_{i}
$$

\subsection{Determination of weight}

In this system, according to different fault degrees, classify gas sensors first, gas sensors $\mathrm{T}_{1}$ and $\mathrm{T}_{2}$ belong to light gas fault and $T_{3}$ and $T_{4}$ belong to heavy gas fault. $T_{1}$ and $T_{2}$ belong to sensors of the same nature and whether gas concentration is over standard or not, their thresholds are the same, after gas concentration is over standard, the system executes the same operation, and therefore we can carry out adaptive weighted data fusion of $T_{1}$ and $T_{2}$ and their weights can be determined by the method of determining weights in adaptive weighted fusion algorithm. For $\mathrm{T}_{3}$ and $\mathrm{T}_{4}$, the actions executed after gas concentration is over standard are the same, but their gas concentration natures are different, so we can not use adaptive weighted data fusion method to carry out data fusion [4]. 


\section{1) Determination of adaptive weighted fusion weights of $T_{1}$ and $T_{2}$ :}

According to adaptive weighted fusion algorithm, the total mean square deviation of a detecting system containing $n$ sensors is

$$
\sigma^{2}=\sum_{i=1}^{n} W_{i}^{2} \sigma_{i}^{2}
$$

In formula (3), $W_{i}$ is weighted factor, namely weight and $\sigma^{2}$ is the multiple quadratic function of $W_{i}$. mean square deviation $\sigma^{2}$ is the least.

$$
W_{i}=\frac{1}{\sigma_{i}^{2} \sum_{i=1}^{n} \frac{1}{\sigma_{i}^{2}}}, \text { The total }
$$

For $\mathrm{T}_{1}$ and $\mathrm{T}_{2}, n=2$. The weights got by calculation are as follows: $W_{\mathrm{T} 1}=0.7366$ and $W_{\mathrm{T} 2}=0.2634$.

\section{2) Determination of weighted data fusion weights of $T_{1}, T_{2}, T_{3}$ and $T_{4}$ :}

Gas concentrations at different underground positions are interactive, the rise of gas concentration at a certain position will inevitably influence gas concentration values at other positions, so choose the fusion weights of $T_{1}, T_{2}, T_{3}$ and $T_{4}$ according to the change law of gas concentration in reality, shown as Table 7 .

Table 7. Fusion Weights of $\mathrm{T}_{1}, \mathrm{~T}_{2}, \mathrm{~T}_{3}$ and $\mathrm{T}_{4}$

\begin{tabular}{cccc}
\hline $\boldsymbol{W}_{\text {T 1 }}$ & $\boldsymbol{W}_{\text {T 2 }}$ & $\boldsymbol{W}_{\text {T 3 }}$ & $\boldsymbol{W}_{\text {T 4 }}$ \\
\hline 0.16 & 0.16 & 0.18 & 0.5 \\
\hline
\end{tabular}

\subsection{Determination of fusion calculation and fault diagnosis threshold}

1) Calculation of adaptive weighted fusion value of $\mathrm{T}_{1}$ and $\mathrm{T}_{2}$ :

Substitute the above calculated mean value $\bar{T}_{i}$ and adaptive weighted weight $W_{\mathrm{T} i}$ of $\mathrm{T}_{1}$ and $\mathrm{T}_{2}$ after errors are eliminated into (1) to get the fusion value of $T_{1}$ and $T_{2}$ is

$$
\begin{aligned}
\hat{T}_{T_{1} T_{2}}=\sum_{i=1}^{2} W_{T i} \bar{T}_{i} & =0.7366 \times 1.3567+0.2634 \times 1.2986=1.3414
\end{aligned}
$$

If the percentage of fusion value of $\mathrm{T}_{1}$ and $\mathrm{T}_{2}$ doesn't exceed $1.5 \%$, it is not over standard and it tallies with the practical situation, which shows the fusion value is valid[5].

2) Calculation of weighted data fusion value of $T_{1}, T_{2}, T_{3}$ and $T_{4}$

Substitute the data in Table 5 and Table 7 into (1) to calculate the fusion value

$$
\begin{aligned}
\hat{T}=\sum_{i=1}^{4} W_{i} T_{i} & =1.3567 \% \times 0.16+1.2986 \% \times 0.16+
\end{aligned}
$$

$1.15 \% \times 0.18+0.44 \% \times 0.5=0.008518$ 
The fusion value in above formula is not the practical meaning of gas concentration and it is only the data judging light and heavy gas.

\section{3) Determination of weighted data fusion and fault diagnosis threshold of $\mathbf{T}_{1}, \mathbf{T}_{2}, \mathbf{T}_{3}$ and $\mathbf{T}_{4}$}

When $\mathrm{T}_{1}, \mathrm{~T}_{2}, \mathrm{~T}_{3}$ and $\mathrm{T}_{4}$ are all at over-standard critical value (0.015), according to (3) and fusion data of $\mathrm{T}_{1}$,

$\mathrm{T}_{2}, \mathrm{~T}_{3}$ and $\mathrm{T}_{4}$ in Table 7 , the fusion result of gas detection data is $\hat{T}=0.00968$. Considering that the maximum divided difference of $\mathrm{T}_{1}, \mathrm{~T}_{2}, \mathrm{~T}_{3}$ and $\mathrm{T}_{4}$ after mistake errors are eliminated in Table $5 \sigma_{2}=0.000297$, and therefore, the fusion threshold of determining that heavy gas fault cuts off local fans and working face is 0.009383 , namely

$$
F_{I}= \begin{cases}\mathbf{1} & \hat{T} \geq 0.009383 \\ \mathbf{0} & \hat{T}<0.009383\end{cases}
$$

In formula (4), $F_{I}=1$ represents the signal that gas fault cuts off local fans and working face.

Similarly, the fusion threshold of determining that light gas fault only cuts off the working face is $0.008904[6]$.

\subsection{Fusion algorithm verification}

According to the mean values of $\mathrm{T}_{1}, \mathrm{~T}_{2}, \mathrm{~T}_{3}$ and $\mathrm{T}_{4}$ in Table 5 after mistake errors are eliminated, fusion weight data of $\mathrm{T}_{1}, \mathrm{~T}_{2}, \mathrm{~T}_{3}$ and $\mathrm{T}_{4}$ in Table 7 , it can be known that the fusion result $\hat{T}=0.008518<0.008904$ is normal.

The calculation result of $(4)$ is $0.009148,0.008904<\hat{T} \quad(=0.009148)<0.009383$, this state belongs to light gas fault.

And then use a set of values after mistake errors are eliminated, $\bar{T}_{1}=0.0148, \overline{T_{2}}=0.0149,{ }^{\bar{T}}=0.015$ and $\overline{T_{4}}=0.0048$, the fusion result got by the same method is 0.009852 . The fusion result $\hat{T}(=0.009852)>$ 0.009383 , this state belongs to heavy gas fault $[3,4]$.

The above data verification proves the validity of fusion algorithm and lays a good foundation for system design.

\section{Conclusion}

This paper mainly studies the fault diagnosis and data processing methods of a double local-fans intelligent monitoring system. Aimed at the practical situation of the system, it carries out related calculations of actual data according to algorithm, including elimination of data containing mistake errors, determination of various weights in data fusion, calculation of fusion value, determination of fault threshold in fault diagnosis and verification of fusion calculation, etc. Through a series of calculations and verifications, it is proved that the data after fusion by this method is more accurate than the result by traditional arithmetic average value method, which can better embody the truth of detection data. In the future, the real-time performance of the system needs to be further improved. 


\section{References}

[1] Chaudhuri, Sam(GEO-Cent, Inc); Crandall, Alan; Reidy, Denis. Multisensor data fusion for mine detecition[A]. Proceedings of SPIE-The Intemation Society for Optical Engineering, p 187-204, Apr 1990.

[2] Vincent Rialle .Laszio Grunfeld .The SCADA (Supervisory Control and Data Acquisition) Monitoring and Control System Based on CAN Bus is Coal Mine .ISTM/2003 .5th Intematinal Symposium on Test and Measurement .Pages 497-500 .Jun 1-5 2003.

[3] Filippidis, Arthur ( Defencs Science Technology Organization) ; Jain , L.C. ; Martin , N.Source.Multisensor data fusion for surface land-mine detection[A] .IEEE Transactions on systems, Man and Cybernetics Part C : Applications and Reviews.P 145-150, Feb 2000.

[4] McCullough, L.R. Application of monitoring and control systems to coal mine ventilation[A. SME Annual Meeting. P 190-194. Feb 26-Mar I 1990.

[5] Aldridge, M. Dayne. Electonic Monitoring and Control of Cal Mine Ventilation. Pap Symp Underground Min $3^{\text {rd }}$, NCA/BCR. P 118-130. 1977.

[6] King, R. H. Eros, L. Microcomputer-based Mine Monitoring System Proves Successful At Dserado Coal Mine. Mining Engineering(Littleton, Colorado). P 40-51. Feb 1988. 